\title{
Placebo found equivalent to amoxicillin for treatment of acute bronchitis in Nairobi, Kenya: a triple blind, randomised, equivalence trial
}

\author{
V N Nduba, ${ }^{1}$ C W Mwachari, ${ }^{1}$ A S Magaret, ${ }^{2}$ D R Park, ${ }^{3}$ A Kigo, ${ }^{1}$ T M Hooton, ${ }^{4}$ \\ C R Cohen ${ }^{5}$
}

${ }^{1}$ Centre for Respiratory Disease Research, Kenya Medical Research Institute, Nairobi, Kenya: ${ }^{2}$ Department of Laboratory Medicine, University of Washington, Seattle, Washington, USA; ${ }^{3}$ Department of Medicine, University of Washington, Seattle, Washington, USA; ${ }^{4}$ Department of Medicine, University of Miami, Miami, Florida, USA; ${ }^{5}$ Department of Obstetrics, Gynecology and Reproductive Sciences, University of California, San Francisco, California, USA

Correspondence to: Dr C R Cohen, Department of Obstetrics, Gynecology and Reproductive Sciences, University of California, San Francisco, 50 Beale Street, Suite 1200, San Francisco, CA 94105 USA; ccohen@ globalhealth.ucsf.edu

Received 7 February 2008 Accepted 14 May 2008 Published Online First 17 June 2008

\section{ABSTRACT}

Background: Antibiotic treatment is not recommended for acute bronchitis in immunocompetent patients in industrialised countries. Whether these recommendations are relevant to the developing world and to immunocompromised patients is unknown.

Design, setting and participants: Randomised, triple blind, placebo controlled equivalence trial of amoxicillin compared with placebo in 660 adults presenting to two outpatient clinics in Nairobi, Kenya, with acute bronchitis but without evidence of chronic lung disease.

Main outcome measure: The primary study end point was clinical cure, as defined by a $\geqslant 75 \%$ reduction in a validated Acute Bronchitis Severity Score by 14 days; analysis was by intention to treat with equivalence defined as $\leqslant 8 \%$ difference between study arms.

Results: Clinical cure rates in the amoxicillin and placebo arms were $81.7 \%$ and $84.0 \%$, respectively (difference $2.3 \%$, $95 \% \mathrm{Cl}-8.6 \%$ to $4.0 \%$ ). Of $131 \mathrm{HIV}$ infected subjects $(19.8 \%)$, cure rates for those randomised to amoxicillin (77.2\%) and placebo (83.8\%) differed by $6.6 \%$ (95\% Cl $-21.7 \%$ to $8.6 \%)$. Among HIV uninfected subjects, the difference in cure rates was $1.6 \%(95 \% \mathrm{Cl}-8.5 \%$ to $5.3 \%)$. Potential drug side effects were similar in the two arms. No subjects required hospitalisation or died.

Conclusion: Antibiotic treatment of acute bronchitis is unhelpful, even in populations with a high prevalence of HIV infection.

Acute bronchitis is the most common respiratory complication experienced by HIV infected persons, particularly in sub-Saharan Africa. In North American and European studies, antibiotic treatment has not been shown to benefit patients with acute bronchitis, ${ }^{1-11}$ and antibiotic treatment is not recommended for this condition in immunocompetent patients in the developed world. ${ }^{12} 13$ Whether these findings and recommendations pertain to immunocompetent and immunocompromised patients in the developing world is unknown. No placebo controlled trials of antibiotic treatment for acute bronchitis in these settings have been published. Nevertheless, the World Health Organization (WHO) has published guidelines recommending that HIV infected patients with acute bronchitis be treated with ampicillin, and rescue treatment with trimethoprimsulfamethoxazole given to those patients who fail to respond. ${ }^{14}$ A Cochrane database review by Fahey et al of randomised controlled trials comparing any antibiotic therapy with placebo in acute bronchitis concluded that antibiotics may have a modest beneficial effect. The benefit seen with antibiotics in the systematic review could be at least in part a result of the anti-inflammatory actions of macrolides and tetracyclines. The magnitude of this benefit, however, was found to be similar to that of the detriment from adverse effects. ${ }^{15}$

In a previous study in Nairobi, Kenya, the WHO approach to the treatment of acute bronchitis in HIV infected patients was prospectively evaluated. Of 401 episodes of acute bronchitis, $97 \%$ appeared to respond clinically to the $1991 \mathrm{WHO}$ recommended antibiotic regimen. ${ }^{16}$ However, we did not test the response rate to placebo alone. Conceivably, as in the case of immunocompetent patients, acute bronchitis may have resolved spontaneously without antibiotic treatment in the majority of HIV infected subjects.

Treatment of acute bronchitis constitutes the most common indication for antibiotic use in the USA and probably in other regions. ${ }^{17}$ This results in high costs, substantial risks of drug side effects and an increased prevalence of antibiotic resistant pathogens. ${ }^{18}$ If antibiotic treatment offers no benefit, it should be withheld in order to minimise these adverse outcomes.

Therefore, we performed a triple blinded, randomised, placebo controlled trial to determine whether amoxicillin and placebo produced equivalent clinical cure rates in the treatment of acute bronchitis in a developing world population with a high prevalence of HIV seropositivity.

\section{METHODS}

The procedures followed were approved by the Kenya Medical Research Institute Ethical Review Committee, the University of Washington Human Subjects Review Committee and the University of California, San Francisco Committee on Human Research, and were in accordance with the ethical standards of the Helsinki Declaration 1975, as revised in 1983 (note that study was begun before clinical trial reporting was mandated). We screened all patients $\geqslant 18$ years old presenting with a productive cough of $\leqslant 2$ weeks duration at the Rhodes Nairobi City Council Clinic between October 2001 and February 2004 and at the Outpatient Department of the Nairobi Mbagathi District Hospital between October 2002 and August 2003. Eligibility was determined by one of two experienced study nurse counsellors previously trained to identify patients who met eligibility criteria. Patients were excluded if they had another potential explanation for cough (history of chronic bronchitis, allergic rhinitis, sinusitis, asthma or 
gastric reflux), serious medical comorbidity (heart disease or diabetes), penicillin allergy, antibiotic use in the preceding 2 weeks or a concurrent infection (including tuberculosis) requiring antibiotic treatment. Otherwise eligible patients were not enrolled if they were unwilling or unable to participate or if they lived outside of Nairobi, making follow-up impractical.

Eligible patients who gave written informed consent were tested for HIV seropositivity using a rapid HIV-1/2 test (Determine, Abbott Park, Illinois USA), with positive results confirmed using a different rapid HIV-1/2 test (Unigold; Trinity Biotech, Bray, Wicklow, Ireland). A posteroanterior chest $x$ ray was performed on all subjects. Those with abnormal chest $x$ ray findings suggesting pneumonia, tuberculosis or other diagnoses were excluded from further participation and were referred for appropriate management.

Subjects were randomly assigned one of two study drugs, amoxicillin $500 \mathrm{mg}$ or placebo, three times daily for 7 days. All study drugs were prepared by the Investigational Drug Services at the University of Washington Medical Center (UWMC), placed in opaque sealed capsules that were identical in appearance, taste and smell, and shipped to the study site in three batches. Doses of 21 capsules were packaged in plastic bottles that were identical except for labels that differed only by a unique identification number. Before leaving the study clinic, each subject was observed to take the first dose of study medication.

Subjects were sequentially assigned a trial number previously allocated to either placebo or amoxicillin (randomised in blocks of 10) by the Investigational Drug Services at the UWMC. The randomisation allocation of amoxicillin or placebo was generated using the Excel (Microsoft, Redman, Washington, USA) random number generator. In addition to subjects, all members of the research team-investigators, physicians, clinical staff, nurse counsellors, research assistants, data collectors, data entry staff and biostatistician-were unaware of the allocation schedule. During the trial the allocation schedule was kept in the office of the Chief Research Pharmacist at UWMC. Masking was maintained until the intention to treat analysis was complete (ie, the analysis was performed by comparing group "A" with group "B").

At enrolment, trained personnel administered a questionnaire addressing sociodemographic information and medical history. The study physician (VN) performed a clinical examination and completed a standardised form that included an assessment of the baseline Acute Bronchitis Severity Score (ABSS). The ABSS measures a spectrum of five patient reported respiratory tract symptoms, ranging from perceived severity of illness to subjective fever (table 1). For each of several symptoms, subjects were asked to gauge severity on a 5 point scale, from non-existent (0) to very serious (4). Validation of the ABSS was carried out during the trial, as previously reported. ${ }^{19}$ During the enrolment visit, sputum was collected for microscopy and culture.

Subjects recruited on Monday, Tuesday and Friday were expected to return for the initial follow-up after 3 days, those enrolled on Thursday were re-evaluated on Monday (4 days) and those recruited on Wednesday were re-evaluated initially on
Friday (2 days). Subjects were also asked to return for a reevaluation after 7 and 14 days. At each visit a standardised questionnaire was re-administered by a research nurse to assess treatment compliance, side effects and current symptoms. The study clinician repeated a physical examination and assessment of the ABSS. Those who were more than 2 days late for any follow-up visit were traced to either their residence or workplace and referred to the clinic for evaluation. A trained field officer performed tracing, with every effort made to maintain the subject's confidentiality. The primary outcome (clinical cure) was defined as a $\geqslant 75 \%$ reduction in baseline ABSS by 14 days. An ABSS at any follow-up visit greater than at enrolment led to open label treatment with erythromycin $500 \mathrm{mg}$ administered orally every $8 \mathrm{~h}$ for 7 days.

\section{Statistical analysis}

We designed the study with sufficient power to evaluate the primary hypothesis: equivalent cure rates for adults treated with amoxicillin or placebo for acute bronchitis. We expected that $85 \%$ of patients with acute bronchitis treated with amoxicillin would be cured. Based on previous equivalency clinical trials and consensus among the investigators, a difference of $8 \%$ was chosen as a reasonable maximal difference in cure rates that could be considered equivalent. For example, any cure rate between $82 \%$ and $98 \%$ in the placebo group compared with a cure rate of $90 \%$ in the amoxicillin group would be defined as equivalent. Using this definition and a two sided test with type I error of $5 \%$, we planned to enrol 335 patients per treatment arm to have $\geqslant 80 \%$ power to conclude equivalence and allow for a $10 \%$ loss to follow-up.

All data were entered using precoded data sheets in TELEform (Cardiff Software, Vista, California, USA). Data were analysed using SPSS for Windows 10.0 (SPSS, Chicago, Illinois, USA). The analysis was performed in an intention to treat manner to evaluate our primary and secondary end points. The primary end point was the difference in clinical cure rates between the amoxicillin and placebo arms using survival analysis (KaplanMeier). Secondary end points included the proportion of subjects experiencing treatment failure, defined as subjects failing to achieve $\mathrm{a} \geqslant 75 \%$ reduction in ABSS from baseline at 2 weeks of follow-up or requiring open label treatment with erythromycin, and the proportion of subjects reporting potential drug side effects.

The Breslow test statistic, appropriate for Kaplan-Meier survival analysis with heavy censoring, was used to compare treatment arms. Ninety-five per cent confidence intervals (CI) were calculated around the difference in cumulative cure rates to determine whether these met our definition for equivalence $( \pm 8 \%)$.

We planned a priori to perform analyses of the primary and secondary end points stratified by HIV serostatus. We evaluated whether treatment success rates differed for HIV infected and uninfected subjects that received amoxicillin versus placebo using survival analysis.

Table 1 Acute Bronchitis Severity Score for acute bronchitis based on 0 to 4 for each of five symptoms (maximum score 20)

\begin{tabular}{llllll}
\hline Symptom & $\mathbf{0}$ & $\mathbf{1}$ & $\mathbf{2}$ & $\mathbf{3}$ & $\mathbf{4}$ \\
\hline Overall severity of illness & Very mild & Mild & Moderate & Serious & Very serious \\
Day cough & $1-2$ times/day & $3-5$ times/day & $6-10$ times/day & $11-20$ times/day & $>20$ times/day \\
Night cough & $1-2$ times/night & $3-5$ times/night & $6-10$ times/night & $11-20$ times/night & $>20$ times/night \\
Limit daily activity & None & Mild & Moderate & Severe & Very severe \\
Subjective fever & None & Mild & Moderate & Severe shaking chills & Very severe \\
\hline
\end{tabular}


Figure 1 Trial profile of a triple blind, randomised, placebo controlled trial of amoxicillin for the treatment of acute bronchitis. CXR, chest $x$ ray.

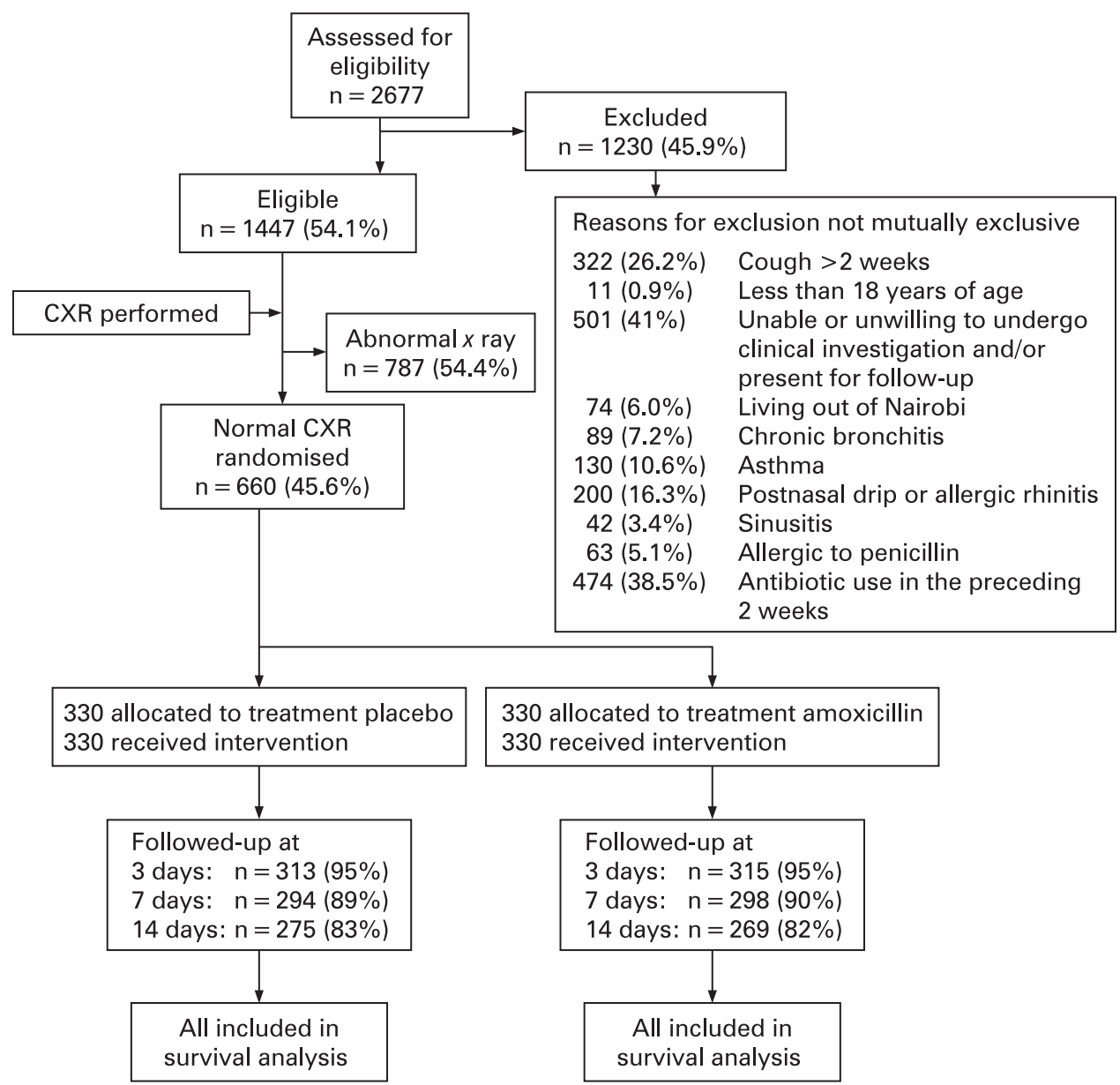

\section{RESULTS}

Of the 2677 subjects with an acute productive cough who were screened for study eligibility, 1230 were ineligible because of one or more exclusion criteria and an additional 787 were excluded because of abnormal chest $x$ ray findings, as interpreted by the study physician; we enrolled 660 subjects (fig 1). Antibiotic use in the past 2 weeks and unwilling to undergo clinical investigation and/or present for follow-up were the two most common reasons for exclusion from enrolment at screening; those willing to participate were similar to those unwilling to participate with respect to mean age (31.2 (9.6) vs 31.0 (9.5) years; $p=0.69)$ and proportion of females ( $56.5 \%$ vs $54.4 \%$; $=0.43$ ).

Table 2 shows the baseline sociodemographic and clinical characteristics of the study participants. The baseline ABSS was normally distributed and had a mean score of 6.3 (3.6) out of 20 (fig 2). Follow-up rates at 3, 7 and 14 days in the two treatment arms were not statistically different, with 275 (83\%) in the placebo arm and 269 (82\%) in the amoxicillin arm completing 14 days of follow-up (fig 1). Ninety-two per cent of subjects enrolled in both arms reported taking $\geqslant 19$ of 21 (90\%) of the study drug capsules $(p=0.96)$. Treatment allocation was not unmasked during the study for any participant. Six hundred and twelve (92.7\%) sputum cultures had no growth. Streptococcus pneumoniae was the most common bacteria detected in sputum ( $n=40,6 \%$ ) followed by Haemophilus influenzae $(\mathrm{n}=5,0.8 \%)$ and Staphylococcus aureus ( $n=3,0.5 \%$ ). Prior to the conduct of this trial, the prevalence of penicillin resistant pneumococci in the study area was $43.3 \%{ }^{20}$

The initial reading of chest $x$ rays was performed by the study physician (VN) at the clinical research site. Subsequently, the chest $x$ rays were reviewed by a consultant radiologist. Of the participants whose initial chest $x$ ray was interpreted as normal, $101(15 \%)$ were subsequently found to have abnormal chest $x$ ray findings, mostly "increased vascular markings" interpreted by the radiologist as suggestive of pneumonitis, with no statistical difference by study arm allocation (40 (12\%) vs 42 $(13 \%))$. According to our study protocol based on concerns for safety, participants with an abnormal chest $x$ ray consistent with pneumonitis were automatically switched to open label treatment with erythromycin at their first follow-up visit. Although the primary intention to treat analysis included all subjects randomised to one of the treatment arms, we also performed a subgroup analysis on the 559 (85\%) subjects with normal chest $x$ rays by the radiologist's reading.

Overall, subjects randomised to amoxicillin and placebo had no significant difference in the cumulative proportion achieving clinical cure by 14 days, $81.7 \%$ and $84.0 \%$, respectively (difference $2.3 \%, 95 \%$ CI $-8.6 \%$ to $4.0 \%$; $=0.40$ ) (fig 3 ). The mean decline in the ABSS score was 4.6 (3.7) and 4.8 (3.8) for the amoxicillin and placebo arms, respectively. The proportion who met our criteria for clinical cure (reduction in ABSS from baseline $\geqslant 75 \%$ ) did not change after limiting the survival analysis to the 559 participants who had normal chest $x$ ray findings confirmed ( $82.2 \%$ vs $84.7 \%$ overall; $p=0.29$ ).

Symptoms that potentially represented adverse events attributable to treatment were reported during follow-up by 183 (58.5\%) and $186(60.4 \%)$ of those who received amoxicillin and placebo, respectively $(p=0.34)$. The number of subjects reporting anorexia (129 (42\%) vs $111(36 \%)$; $\mathrm{p}=0.12)$, nausea $(92(30 \%)$ vs $94(30 \%)$; $\mathrm{p}=1.0)$, vomiting $(17(6 \%)$ vs $18(6 \%) ; \mathrm{p}=1.0)$, abdominal pain (74 (24\%) vs 80 (26\%), $p=0.71$ ) and diarrhoea (26 (8\%) vs 34 
Table 2 Baseline sociodemographic and clinical characteristics of the trial groups

\begin{tabular}{|c|c|c|c|}
\hline Characteristic & $\begin{array}{l}\text { Placebo } \\
(\mathrm{n}=330)\end{array}$ & $\begin{array}{l}\text { Amoxicillin } \\
(\mathrm{n}=330)\end{array}$ & p Value \\
\hline \multicolumn{4}{|l|}{ Sociodemographic } \\
\hline Age (y) (mean (SD)) & $29.7(9.4)$ & $29.8(8.8)$ & 0.94 \\
\hline Female & $205(62.1)$ & 195 (59.1) & 0.38 \\
\hline HIV infected & $66 / 330(20.0)^{*}$ & $65 / 329(19.8)$ & 0.94 \\
\hline CD4 counts $(25,75$ percentiles) & $303(119,516)$ & $367(197,530)$ & \\
\hline Cigarette smoker & $38 / 323(11.8)$ & $40 / 326(12.3)$ & 0.90 \\
\hline \multicolumn{4}{|l|}{ Marital status } \\
\hline Single & 109/328 (33.2) & $114 / 330(34.5)$ & \\
\hline Married/cohabiting & $202 / 328(61.6)$ & $189 / 330(57.3)$ & \\
\hline Divorced/separated/widowed & $17 / 328(5.2)$ & $27 / 330(8.2)$ & 0.25 \\
\hline Employed & $215 / 327(65.7)$ & $235 / 330(71.2)$ & 0.15 \\
\hline Has savings & $103 / 327(31.5)$ & $108 / 329(32.8)$ & 0.74 \\
\hline Education $\leqslant 8$ y & $164 / 327(50.2)$ & $160 / 330(48.5)$ & 0.70 \\
\hline \multicolumn{4}{|l|}{ Symptoms } \\
\hline Shortness of breath & 108/323 (33.4) & 106/326 (32.5) & 0.80 \\
\hline Chest pain & $240 / 323(74.3)$ & $237 / 325(72.9)$ & 0.72 \\
\hline Wheezing & $149 / 323(46.1)$ & $134 / 326(41.1)$ & 0.21 \\
\hline Duration of cough (days) (mean (SD)) & $8.3(4.3)$ & $8.4(4.4)$ & 0.78 \\
\hline Anorexia & 109/323 (33.7) & $117 / 326(35.9)$ & 0.62 \\
\hline Nausea & $58 / 323(18.0)$ & $73 / 326(22.4)$ & 0.20 \\
\hline Vomiting & $21 / 323(6.5)$ & $21 / 326(6.4)$ & 0.87 \\
\hline Abdominal pain & $58 / 323(18.0)$ & $59 / 326(18.1)$ & 1.0 \\
\hline Diarrhoea & $14 / 323(4.3)$ & $17 / 326(5.2)$ & 0.71 \\
\hline
\end{tabular}

Values are number (\%) unless otherwise stated.

*Relevant denominator indicated.

$(11 \%) ; p=0.34)$ were not statistically different in the amoxicillin and placebo arms of the study, respectively.

We performed an intention to treat analysis of all participants, most of whom were unaware of their HIV serostatus at the time of presentation. An a priori goal of this study was to perform an analysis of clinical cure stratified by HIV serostatus. No significant differences were found between the amoxicillin and placebo arms for either HIV infected ( $p=0.94)$ (fig 4) or HIV uninfected subjects $(p=0.35)$ (table 3$)$.

Our a priori definition of equivalent clinical cure between study arms was an outcome difference of $\leqslant 8 \%$. To more easily

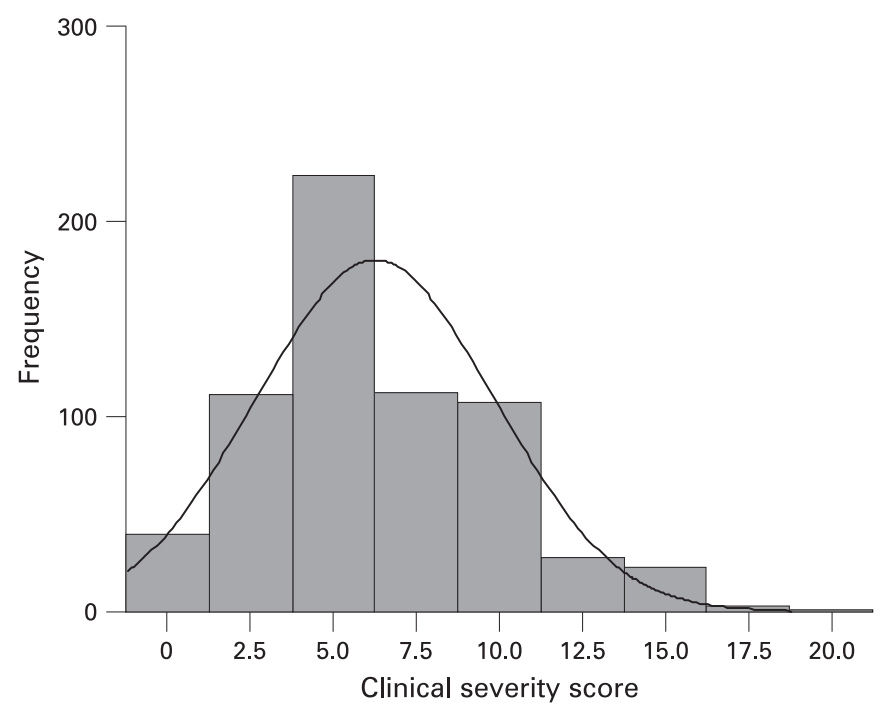

Figure 2 Distribution of baseline Acute Clinical Severity Score used to determine the severity of acute bronchitis in 660 enrolled cases (mean $11.0(5.1))$. interpret our findings in the context of an equivalence trial, we calculated the difference in clinical cure with corresponding $95 \%$ CI between the study arms. Table 3 demonstrates these results for the overall study population, for subjects with a confirmed normal chest $x$ ray and for HIV infected and uninfected participants. For the overall study population, the difference in cure rate was $2.3 \%(95 \% \mathrm{CI}-8.6 \%$ to $4.0 \%$; meaning that in $95 \%$ of identically designed trials in the same population, the effect of amoxicillin would fall within $8.6 \%$ worse and $4.0 \%$

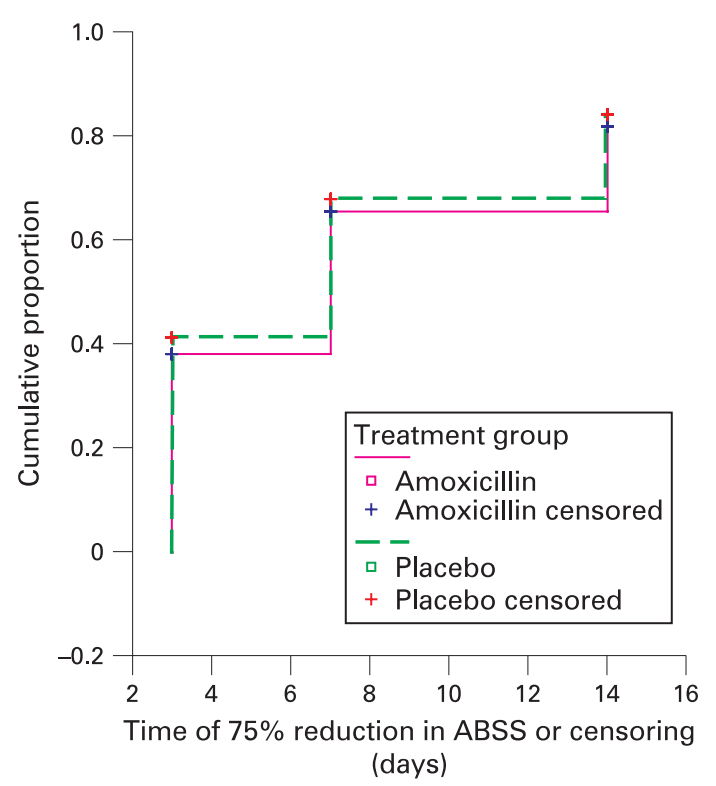

Figure 3 Cumulative proportion clinically cured, as defined by $a \geqslant 75 \%$ reduction in baseline clinical severity score (in days), by treatment group (see fig 1 for numbers censored at 3, 7 and 14 days because of dropouts). ABSS, Acute Bronchitis Severity Score. 


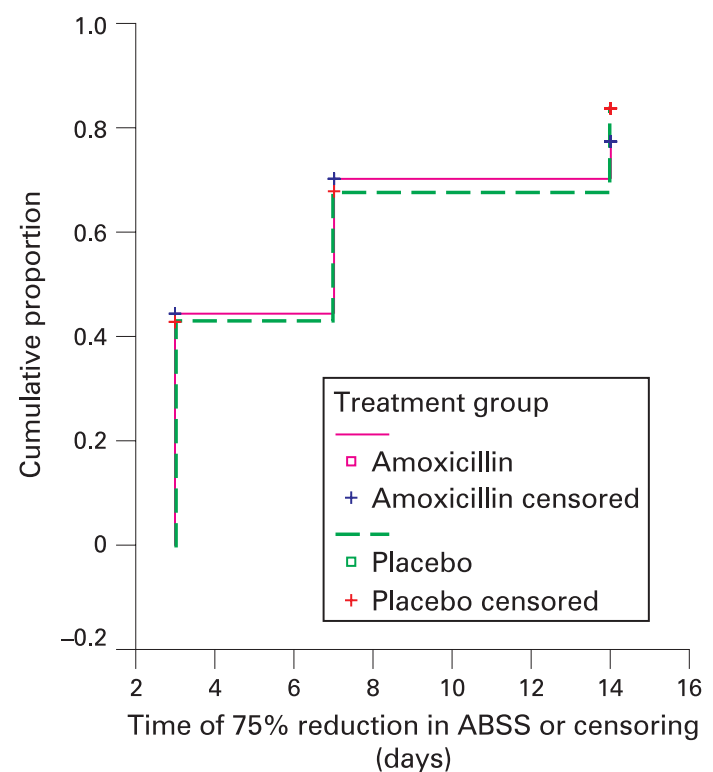

Figure 4 Among HIV infected participants, cumulative proportion clinically cured, as defined by a $\geqslant 75 \%$ reduction in clinical severity score (in days), by treatment group (numbers censored at 3, 7 and 14 days were 10, 17 and 33, respectively). ABSS, Acute Bronchitis Severity Score.

better than that of placebo). Thus we had $95 \%$ confidence that the confidence limits only slightly exceeded our preselected definition of equivalence $( \pm 8 \%)$. The difference and confidence limits were similar when the analysis was limited to include only those with a confirmed normal chest $x$ ray and for HIV seronegative participants. The difference in outcomes between study arms in the HIV infected group was small (6.6\%), but the $95 \%$ confidence limits were wider $(-21.7 \%$ to $8.6 \%)$ because of the smaller numbers and limited statistical power. (Note that the point estimate favours placebo in every comparison.) Further stratified analysis of the HIV infected subgroup using CD4 counts as a covariate did not change the outcome; similar findings were found when limiting the analysis to the 559 subjects with normal chest radiographic findings (data not shown).

\section{DISCUSSION}

Our study is the largest placebo controlled trial of antibiotic treatment of acute bronchitis that has been reported to date. ${ }^{1-468-11}$ It is the first to study patients in the developing world, and the first to include HIV infected subjects. Notably, ours is also the first study of acute bronchitis treatment to use an equivalence design - intended and powered to prove equivalent treatment effects as opposed to the lack of a significant difference in effects.
As an equivalence trial, this study is distinct from previous randomised controlled trials of acute bronchitis, which tested for superiority of an antibiotic over placebo. ${ }^{1-4} 6$ 8-11 $^{-1}$ Superiority trials that fail to demonstrate a statistical difference between two interventions, or an intervention and a placebo, should not be misinterpreted as demonstrating equivalence when in fact they are underpowered to do so within the clinically relevant margins. ${ }^{21}$ Interpretation of equivalence trials requires evaluation of confidence intervals for the differences in treatment outcomes and the clinical importance of such differences, rather than statistical significance. ${ }^{22}$

Determination of what constitutes a clinically significant difference is an essential part of the design of an equivalence trial, but this is a necessarily arbitrary distinction. In computing the sample size for our study, we arbitrarily defined $\pm 8 \%$ to be the minimum treatment difference that would be considered clinically significant. The $95 \%$ CI for the difference in treatment effects in our study ranged from $8.6 \%$ worse cure rate to a $4.0 \%$ better cure rate with amoxicillin in comparison with placebo. This range is within our a priori criteria for equivalence at the upper level As a result, we are $95 \%$ confident that the outcomes of amoxicillin treated patients were $\leqslant 4.0 \%$ better than those of patients given placebo.

The burden of respiratory tract infection (RTI) is far greater in sub-Saharan Africa than in the US and Europe, ${ }^{1623}$ and sociodemographic and behavioural risk factors for acute bronchitis that may affect aetiology and clinical course differ between developed and developing regions (eg, cigarette smoking vs poverty factors, respectively) ${ }^{24}$ Thus findings of studies in North America and Europe may not be applicable to sub-Saharan Africa and other developing regions. In this context, it is reassuring to find that our results agree with those of earlier investigations, including a recently published paper that did not find any benefit of azithromycin over low dose vitamin C. ${ }^{3}$

Our study measured disease severity using a predefined, easily replicable ABSS composed of RTI related symptoms, as well as an a priori definition of clinical cure. We chose to develop our ABSS because of the lack of published disease specific severity scoring systems for patients with acute bronchitis. This newly developed score proved reliable, valid and highly responsive. ${ }^{19}$ The only other acute bronchitis study that has used a disease specific symptom scoring system was published after the design and onset of our study. ${ }^{3}$

Our inclusion of HIV infected subjects is another important contribution. Acute bronchitis arguably may be the most common HIV associated opportunistic infection. ${ }^{23}$ Although antibiotic treatment is recommended by the WHO for HIV infected subjects with acute bronchitis, ${ }^{14}$ whether antibiotic treatment results in any benefit is unknown. To address this issue, we chose to enrol all subjects with acute bronchitis and then to stratify our analyses based on HIV serostatus. This reflects the real world situation in which diagnosis and

Table 3 Clinical cure estimates and confidence intervals in the two treatment arms

\begin{tabular}{|c|c|c|c|c|c|c|c|c|}
\hline & \multicolumn{2}{|c|}{$\begin{array}{l}\text { All subjects } \\
\text { included } \\
(\mathrm{n}=660)\end{array}$} & \multicolumn{2}{|c|}{$\begin{array}{l}\text { Subjects with } \\
\text { normal } \\
x \text { ray* }(n=559)\end{array}$} & \multicolumn{2}{|c|}{$\begin{array}{l}\text { HIV+/ve } \\
(n=131)\end{array}$} & \multicolumn{2}{|l|}{$\begin{array}{l}\text { HIV-/ve } \\
(\mathrm{n}=529)\end{array}$} \\
\hline & Placebo & Amoxicillin & Placebo & Amoxicillin & Placebo & Amoxicillin & Placebo & Amoxicillin \\
\hline $\begin{array}{l}\text { Cumulative proportion } \\
\text { cured }\end{array}$ & $84.0 \%$ & $81.7 \%$ & $84.7 \%$ & $82.2 \%$ & $83.8 \%$ & $77.2 \%$ & $84.0 \%$ & $82.4 \%$ \\
\hline Difference in cure rates & \multicolumn{2}{|l|}{$2.3 \%$} & \multicolumn{2}{|l|}{$2.5 \%$} & \multicolumn{2}{|l|}{$6.6 \%$} & \multicolumn{2}{|l|}{$1.6 \%$} \\
\hline $95 \% \mathrm{Cl}$ for difference & \multicolumn{2}{|c|}{$-8.6 \%$ to $4.0 \%$} & \multicolumn{2}{|c|}{$-9.2 \%$ to $4.2 \%$} & \multicolumn{2}{|c|}{$-21.7 \%$ to $8.6 \%$} & \multicolumn{2}{|c|}{$-8.5 \%$ to $5.3 \%$} \\
\hline
\end{tabular}

${ }^{*}$ After enrolment and randomisation, 101 subjects were determined to have an abnormal chest $x$ ray by the consultant radiologist, leaving 559 evaluable participants. 
treatment decisions often must be made in advance of awareness of an individual patient's HIV serostatus. In our study, HIV infection did not affect the resolution of acute bronchitis. Furthermore, separate analysis of HIV infected and uninfected participants demonstrated similar responses for those randomised to amoxicillin and placebo. However, this study was not powered to unequivocally establish equivalence in the HIV infected subgroup; comparing amoxicillin with placebo, the $95 \% \mathrm{CI}$ $(-21.7 \%$ to $8.6 \%)$ extends slightly beyond our predefined criteria for a clinically important treatment difference. An adequately powered randomised placebo controlled equivalence trial of antibiotic treatment for acute bronchitis in HIV infected adults would be needed to definitively settle this question.

Our study had several limitations. The lack of a substantial effect of antibiotic treatment might be attributed to the use of the wrong antibiotic. We chose to use amoxicillin rather than a newer antibiotic because it is affordable, widely available and prescribed and, most importantly, was recommended by the WHO for the management of acute bronchitis in HIV infected patients. ${ }^{14}$ The lack of an effect of azithromycin in another recent trial further supports the notion that antibiotic therapy, in general, offers little benefit for the treatment of acute bronchitis. ${ }^{3}$ The argument against antibiotics for treatment of acute bronchitis is further reinforced by the Cochrane review by Fahey et al which concluded that the magnitude of benefit of antibiotics was similar to detriments from adverse effects. ${ }^{15}$

Fifteen per cent of randomised subjects had abnormal chest $x$ rays identified by the consultant radiologist after randomisation. In most of the cases, the finding was "prominent vascular markings" that may have represented pneumonitis, or may have been unrelated to the acute respiratory illness. In any case, for safety reasons, these subjects were withdrawn from the randomised treatment trial and given open label erythromycin at the next follow-up visit (usually day 3 ). This delay in receiving treatment may have inadvertently increased the rate of treatment failure in both arms. However, a limitation of the analysis to those with confirmed normal chest radiographs did not affect our study outcome. Our defaulter rate was similar in both study arms; $17.6 \%$ of study participants did not complete the full evaluation of the study outcomes. Unfortunately, we cannot determine whether those lost to follow-up improved, worsened or remained clinically unchanged.

Several additional factors related to selection of participants may limit the external validity of our study and therefore require further discussion. Although most patients diagnosed with acute bronchitis do not have a chest $x$ ray performed, we excluded those with abnormal radiographic findings to avoid delaying treatment of individuals with pneumonia and tuberculosis. Future investigations should prospectively measure the predictive values of clinical algorithms to diagnose pulmonary infiltrates in populations with high HIV seroprevalence, a method found moderately useful in studies conducted in the USA. ${ }^{25}{ }^{26}$ For obvious reasons, we excluded patients unwilling to participate in the study. Although those unwilling to participate were more likely to report another exclusion criterion such as recent antibiotic use and duration of cough $\geqslant 2$ weeks than those willing to participate, the analysis revealed no major source of selection bias that could seriously invalidate the generalisability of the study results.

This study was conducted in cooperation with the Integrated Management of Adolescent and Adult Illness (IMAI), part of the 3 by 5 Initiative at the WHO. ${ }^{27}$ As such these data have provided necessary evidence to help revise the IMAI Acute Care Module for first level facility health workers in resource poor settings. ${ }^{27}$ Results from this and other trials need to influence not only treatment guidelines, but also patient awareness, ${ }^{12}$ in order to guide more responsible antibiotic use in the treatment of RTIs.

Acknowledgements: The authors would like to acknowledge Maryanne Munene, Naomi Mwachari, Pamela Omuom, Rose Wanjala and Jackson Achando who collected these data and most importantly cared for the study participants, Elizabeth Mwachari for volunteering to perform data management, the Director KEMRI for allowing the performance of this investigation and most of all the study participants themselves. VND implemented the study, collected the data, performed the analysis and wrote the manuscript. CM designed the protocol and supervised study implementation, analysis of the data and writing of the manuscript. ASM advised on study design, supervised data analysis and assisted with manuscript preparation. DRP helped oversee study implementation, and supervised data analysis and writing of the manuscript. AK assisted with study implementation, and oversaw the radiographic elements of the study. TH helped with study design and review of the manuscript. CC designed the protocol and supervised study implementation, analysis of the data and writing of the manuscript.

Funding: This study was supported by a grant from the Rockefeller Foundation. VNN was a fellow in the International AIDS Research and Training Program at the University of Washington supported by the Fogarty International Center (T22TW00001). The Rockefeller Foundation did not participate in the development of the study design, implementation or analysis of the data, or in the decision to submit this manuscript for publication.

\section{Competing interests: None.}

Ethics approval: The procedures followed were approved by the Kenya Medical Research Institute Ethical Review Committee, the University of Washington Human Subjects Review Committee and the University of California, San Francisco Committee on Human Research.

\section{REFERENCES}

1. Brickfield FX, Carter WH, Johnson RE. Erythromycin in the treatment of acute bronchitis in a community practice. J Fam Pract 1986;23:119-22.

2. Dunlay J, Reinhardt R, Roi LD. A placebo-controlled, double-blind trial of erythromycin in adults with acute bronchitis. J Fam Pract 1987;25:137-41.

3. Evans AT, Husain S, Durairaj L, et al. Azithromycin for acute bronchitis: a randomised, double-blind, controlled trial. Lancet 2002;359(9318):1648-54.

4. Franks $\mathbf{P}$, Gleiner JA. The treatment of acute bronchitis with trimethoprim and sulfamethoxazole. J Fam Pract 1984;19:185-90.

5. Hueston WJ. A comparison of albuterol and erythromycin for the treatment of acute bronchitis. J Fam Pract 1991;33:476-80.

6. King DE, Williams WC, Bishop L, et al. Effectiveness of erythromycin in the treatment of acute bronchitis. J Fam Pract 1996;42:601-5.

7. Littenberg B, Wheeler M, Smith DS. A randomized controlled trial of oral albuterol in acute cough. J Fam Pract 1996;42:49-53.

8. Scherl ER, Riegler SL, Cooper JK. Doxycycline in acute bronchitis: a randomized double-blind trial. J Ky Med Assoc 1987;85:539-41.

9. Stott NC, West RR. Randomised controlled trial of antibiotics in patients with cough and purulent sputum. Br Med J 1976;2:556-9

10. Verheij TJ, Hermans J, Mulder JD. Effects of doxycycline in patients with acute cough and purulent sputum: a double blind placebo controlled trial. Br J Gen Pract 1994;44:400-4.

11. Williamson HA Jr. A randomized, controlled trial of doxycycline in the treatment of acute bronchitis. J Fam Pract 1984;19:481-6.

12. Gonzales R, Camargo CA Jr, MacKenzie T, et al. Principles of appropriate antibiotic use for treatment of uncomplicated acute bronchitis: background. Ann Intern Med 2001;134:521-9.

13. Gonzales R, Sande MA. Uncomplicated acute bronchitis. Ann Intern Med 2000; 133:981-91.

14. WHO. Guidelines for the clinical management of HIV infections in adults. Geneva: WHO/GPA, 1991.

15. Fahey TSJ, Becker L, Glazier R. Antibiotics for acute bronchitis. Cochrane Database Syst Rev 2004;4:CD000245.

16. Mwachari CW, Cohen CR, Meier AS, et al. Respiratory tract infection in HIV-1 infected adults in Nairobi, Kenya: evaluation of risk factors and the world health organization treatment algorithm. J Acquir Immune Defic Syndr 2001;27:365-71.

17. Gonzales R, Malone DC, Maselli JH, et al. Excessive antibiotic use for acute respiratory infections in the United States. Clin Infect Dis 2001;33:757-62.

18. Wise RHT, Hart T, Cars 0 , et al. Antimicrobial resistance. Is a major threat to public health. BMJ 1998;317:609-10.

19. Mwachari CWNV, Nguti R, Park DR, et al. Validation of a new clinical scoring system for acute bronchitis. Int J Tuberc Lung Dis 2007;11:1253-9.

20. Kariuki S, Muyodi J, Mirza B, et al. Antimicrobial susceptibility in communityacquired bacterial pneumonia in adults. East Afr Med J 2003;80:213-17.

21. Piaggio GPA. Use of the equivalence approach in reproductive health clinical trials Stat Med 2001;20:3571-7. 
22. Fleming TRLD. Survival analysis in clinical trials: past developments and future directions. Biometrics 2000;56:971-83.

23. Mwachari CWSB, Cleopa 0, Odhiambo JA, et al. Mortality and burden of disease in a cohort of HIV-seropositive adults in Nairobi, Kenya. Int J STD AIDS 2004:15:120-6.

24. Penner JMA, Meier AS, Mwachari $\mathrm{C}$, et al. Risk factors for pneumonia in urbandwelling HIV-infected women: a case-control study in Nairobi, Kenya. J Acquir Immune Defic Syndr 2003;32:2.
25. Heckerling PSGB, Tape TG, Wigton RS. Prediction of community-acquired pneumonia using artificial neural networks. Med Decis Making 2003;23:112-21.

26. Heckerling PSTT, Wigton RS, et al. Clinical prediction rule for pulmonary infiltrates Ann Intern Med 1990:113:664-70.

27. WHO. Acute care: interim guideline for first level facility health workers. Integrated management of adolescent and adults illness. http://www.who.int/3by5/publications/ documents/en/MAl Acutecare.pdf (accessed 8 August 2008).

\section{Pulmonary puzzle}

\section{ANSWER}

From the question on page 980

The patient underwent a thoracotomy with wedge resection of the right upper lobe of the lung and pathological examination of a specimen showed the presence of malignancy. Lobectomy of the right upper lobe was performed. Microscopic examination revealed sarcomatous tumour cells with dyscohesive pleomorphic nuclei and prominent infiltration (fig 1A). Upon immunochemical analysis the tumour cells were positive for cytokeratin and vimentin (fig $1 \mathrm{~B}$ and $\mathrm{C}$ ). The tumour was compatible with a giant cell carcinoma. Thyroid transcription factor-1 was measured to confirm the primary lung origin.

The patient developed intussusception due to intestinal metastasis 2 weeks later (fig 1D) and underwent segmental resection of the small intestine. Postoperative adjuvant chemotherapy was advised but the patient refused and he died 6 months later.

Giant cell carcinoma is classed as a pleomorphic carcinoma according to the World Health Organization classification. ${ }^{1}$ It is a group of poorly differentiated non-small cell lung cancers (NSCLCs) that have sarcomatous or sarcoma-like elements. Examples of pleomorphic carcinoma are NSCLC; squamous cell carcinoma; adenocarcinoma; large-cell carcinoma containing spindle cells, giant cells, or both; or a carcinoma consisting only of spindle cells and giant cells. Most tumours with two cell components can be classified as pleomorphic carcinomas, but the pure spindle or giant cell tumour had also been reported. In the present case, we called it "giant cell carcinoma" because the tumour contained only the giant cell.

The incidence of pulmonary pleomorphic carcinoma is low (1.6\% of resected NSCLCs. ${ }^{2}$ Pulmonary pleomorphic carcinoma presented in a symptomatic male smoker as a large peripheral lesion. Patients present with tumour-related symptoms such as chest pain, coughing and haemoptysis. ${ }^{3}$ These tumours are more aggressive and result in significantly poorer survival than other NSCLCs. ${ }^{4}$ The biphasic appearance of the tumour and its rarity often make diagnosis difficult.

Pure giant cell tumours of the lung are extremely uncommon. We have described an atypical image of a giant cell carcinoma of the lung. Positron emission tomography (PET) is useful for determining the distal metastases and assessment of mediastinal lymph nodes. As might be expected, intestinal metastasis may be detected if the PET is performed preoperatively. Surgical resection and aggressive postoperative chemotherapy should be considered in such cases.

Thorax 2008;63:1005. doi:10.1136/thx.2007.091751a

\section{REFERENCES}

1. Yuki T, Sakuma T, Ohbayashi $C$, et al. Pleomorphic carcinoma of the lung: a surgical outcome. J Thorac Cardiovasc Surg 134:2:399-404.

2. Rossi G, Cavazza A, Sturm N, et al. Pulmonary carcinomas with pleomorphic, sarcomatoid or sarcomatous elements: a clinicopathologic and immunohistochemical study of 75 cases. Am J Surg Pathol 2003;27:311-24.

3. Raveglia F, Mezzetti M, Panigalli T, et al. Personal experience in surgical management of pulmonary pleomorphic carcinoma. Ann Thorac Surg 2004;78:1742-7.

4. Fishback NF, Travis WD, Moran CA, et al. Pleomorphic (spindle/giant cell) carcinoma of the lung. A clinicopathologic correlation of 78 cases. Cancer 1994;73:2936-45.
Figure 1 (A) Sarcomatous tumour cells showing dyscohesive pleomorphic nuclei and prominent nucleoli in diffuse infiltration with extensive necrosis (H\&E stain, $400 \times)$. Tumour cells stained positively for (B) cytokeratin and (C) vimentin. (D) CT scan of the abdomen demonstrating nodule target lesion (arrow) over the right lower abdomen and intestinal dilatation, which was consistent with an intestinal intussusception.
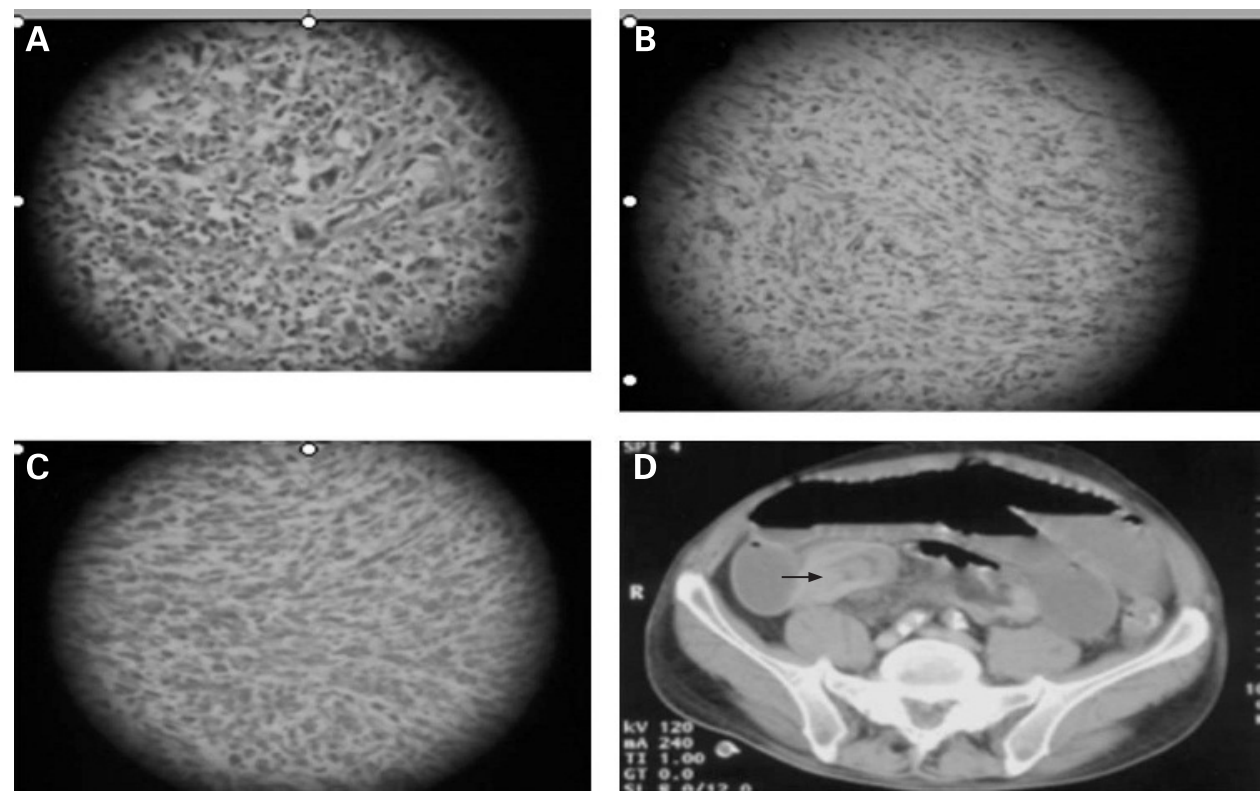